\title{
Medición de las capacidades tecnológicas en la industria mexicana
}

\author{
Lilia Dominguez y Flor Brown
}

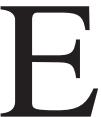

ste trabajo realiza una aportación metodológica y analítica a la línea de investigaciones abocadas a construir índices representativos de las capacidades tecnológicas de las empresas manufactureras mexicanas. Asimismo, examina la distribución de estas capacidades en una muestra de estos establecimientos y su asociación con variables de desempeño con base en una muestra de 1.818 empresas. Mediante el análisis factorial se identificaron cuatro factores que expresan las principales fuentes de aprendizaje en la empresa manufacturera: i) política de formación de personal, ii) innovación de mejora continua, iii) sistemas de información y documentación, y iv) inversión en nuevas tecnologías. El análisis de agrupamientos permitió identificar cuatro grupos de empresas en función de los puntos obtenidos por factor y examinar sus indicadores de desempeño. Se observó que hay una asociación positiva ces: el margen de ganancia, la productividad laboral y el cambio técnico. 


\section{I}

\section{Introducción}

Hay creciente acuerdo en que la heterogeneidad de las empresas de los países en desarrollo tiene importantes efectos. Este trabajo postula que el análisis de los niveles de las capacidades tecnológicas de las empresas aporta elementos para comprender los orígenes de las diferencias que ellas presentan. Tales diferencias desempeñan un papel central en la teoría del proceso evolutivo, que destaca la trascendencia del aprendizaje en la generación de cambio técnico (Nelson y Winter, 1982). Según dicha teoría, los individuos, las empresas y las economías nacionales crearán riqueza y lograrán tener acceso a la riqueza de acuerdo con su capacidad de aprendizaje (Lundvall, 1992). Se cuestiona en ella la visión simplista que reduce el significado de la tecnología a máquinas o manuales que indican los pasos que han de seguirse. Por el contrario, la tecnología implica ante todo transmisión de conocimiento entre distintos agentes; se caracteriza por tener componentes tácitos de conocimiento específico tanto de las personas como de las prácticas internas de la empresa que la genera; y se desarrolla a partir de los procedimientos de búsqueda y aprendizaje para mejorar la eficiencia productiva, generar nuevos productos e introducir métodos de organización. En oposición a los enfoques tradicionales, se señala que el desarrollo tecnológico industrial no debe verse como un proceso que sólo se puede promover por medio de la inversión en nuevos equipos y la compra de tecnología importada. Para que la tecnología pueda ser asimilada, bien operada y mejorada, las empresas deben realizar inversiones y acciones deliberadas de aprendizaje tecnológico. Por lo tanto, no basta con comprar máquinas o contratar transferencia de tecnología para reducir la brecha tecnológica internacional. Las empresas deben investigar la tecnología, entenderla y documentarla para asimilarla y mejorarla.

En el mismo sentido, Bell y Pavitt (1993) ponen en duda la distinción entre innovación y difusión. Señalan que la difusión implica mucho más que la adquisición de maquinaria y el conocimiento técnico conexo, pues significa también cambio técnico continuo y a menudo incremental, para adecuarse a situaciones específicas y alcanzar mejores estándares de desempeño. Distinguen tres etapas en la dinámica del cambio técnico en los países en desarrollo: en la pri- mera se adopta tecnología con la incorporación de nuevas instalaciones y se adapta o mejora la tecnología original para adecuarla a la situación específica; en la segunda se busca elevar la eficiencia inicial y se modifica la tecnología para responder a los cambios en los mercados de insumos y productos. Las dos etapas descritas requieren una continua acumulación de conocimiento y habilidades. En la tercera etapa, las empresas pueden basarse en el conocimiento, la experiencia y las nuevas destrezas adquiridas para introducir un cambio técnico más sustancial. Los autores citados definen las capacidades tecnológicas como las habilidades necesarias para generar y administrar el cambio técnico, que incluyen destrezas, conocimientos y experiencias distintas de las requeridas para operar los sistemas técnicos.

Numerosos estudios muestran que la capacidad de aprendizaje y la acumulación de capacidades tecnológicas están detrás de la competitividad de los países altamente desarrollados (Lundvall, 1992). Asimismo, se ha comprobado que el éxito exportador de las empresas sudasiáticas, particularmente coreanas y taiwanesas, se ha debido no sólo a esquemas de políticas de mercado o importación de tecnología, sino también al hecho de que éstos fueron acompañados por un conjunto de acciones estratégicas destinado a incrementar la capacidad de aprendizaje de esas empresas (Pack y Westphal, 1986; Fransman, 1984; Amsden, 1992). Respecto del caso latinoamericano existen ya varios estudios que muestran, para algunos sectores industriales, las modalidades de aprendizaje tecnológico de las empresas y sus efectos en la modernización (Katz, 1997 y 1987). Por su parte, Cimoli (2000) analizó diversos aspectos del sistema nacional de innovación en la industria mexicana.

La evidencia proporcionada por estos trabajos es valiosa, ya que ellos examinaron por primera vez las condiciones necesarias para la innovación desde una perspectiva sistémica, rica en detalles. Gracias a ellos se cuenta con un diagnóstico de las empresas que más influyen en la economía y que constituyen uno de los pilares del sistema nacional de innovación. Sin embargo, aunque hay estudios sobre la construcción de indicadores con distintas fuentes y metodología, el avance ha sido menor en la construcción de indicadores 
por empresa que provengan de una muestra de tamaño significativo; de esta manera se podría esclarecer en forma precisa el nivel de acumulación de las capacidades tecnológicas. ${ }^{1}$ Estos indicadores de las capacidades tecnológicas de las empresas pueden contribuir a establecer, en forma más fina que en los estudios de casos, los factores que subyacen las diferencias entre empresas y los efectos que ellos tienen en su desempeño.

Este trabajo aspira a avanzar por el mismo cauce, es decir, a elaborar indicadores que permitan medir las capacidades tecnológicas en la industria mexicana. ${ }^{2}$ El propósito es dilucidar las actividades de aprendizaje que más inciden en la capacidad para administrar y generar el cambio técnico en las empresas manufactureras mexicanas. El reto radica en la dificultad de captar en toda su complejidad el concepto de capacidades tecnológicas, que abarca muchos aspectos. Esto lleva a utilizar algún método para avanzar hacia su relativa simplificación. Creemos que la técnica estadística apropiada es el análisis factorial, que puede representar relaciones entre conjuntos de variables interrelacionadas y explicar conceptos complejos sin imponer previamente una estructura. La finalidad es proveer herramientas para el análisis de las capacidades tecnológicas por medio de uno o varios índices, a partir de los aportes de Bell y Pavitt (1992) y de Lall (1992). ${ }^{3}$

Un segundo objetivo de esta investigación es identificar grupos de empresas por niveles de capacidades tecnológicas, con la intención de analizar hasta qué punto los niveles de cada grupo se relacionan con diferencias significativas en el desempeño.

Este trabajo consta de cinco secciones. Tras esta introducción (sección I), se hace una somera revisión de estudios recientes sobre la construcción de índices tecnológicos (sección II). Se describe la metodología que se utilizó para elaborar los índices de capacidades tecnológicas (sección III). Se analizan dichos índices, así como las características de los grupos de empresas industriales que se elaboraron sobre la base de los índices calculados, y se presentan algunas medidas de desempeño de grupos con distintos niveles de capacidades tecnológicas (sección IV). Por último, en la sección $\mathrm{V}$ se presentan las conclusiones.

\section{II}

\section{Construcción de indicadores o índices}

\section{tecnológicos: contribuciones recientes}

En esta sección pasamos revista a algunos de los estudios más influyentes sobre el tema. En ellos se encuentran distintas formas de cuantificar las capacidades tecnológicas de las empresas, así como estimaciones de índices compuestos. Buena parte de esos estudios utiliza las aportaciones de Bell y Pavitt (1992) y, en especial, la taxonomía de Lall (1992), quien sugiere formas de clasificar las capacidades tecnológicas desarrolladas por la empresa con el fin de asimilar, adaptar y mejorar la tecnología adquirida. La taxonomía mencionada distingue capacidades de inversión, de producción y de vinculación. Para Lall, las capacidades de inversión son las habilidades necesarias para

\footnotetext{
${ }^{1}$ Autores como Tremblay (1998) y Romijn (1999) han hecho presente la necesidad de indicadores robustos.

${ }^{2}$ Agradecemos la participación del Instituto Nacional de Estadística, Geografía e Informática (INEGI) en la estimación por establecimiento.
}

identificar, preparar y obtener tecnología para el diseño, la construcción, el equipamiento y el personal de un nuevo proyecto. Los costos de capital del proyecto dependen de la escala de producción, la composición del conjunto de bienes producidos, la selección de tecnología y la comprensión que tenga la empresa de las tecnologías involucradas. Las capacidades de producción van desde las habilidades básicas (control de calidad, operación, mantenimiento) hasta las más avanzadas (adaptación, mejora) y las más exigentes (investigación, diseño, innovación). Estas habilidades permiten no sólo operar y mejorar tecnologías, sino también efectuar esfuerzos internos para absorber o imitar la tecnología comprada a otras empresas. Las capacidades de vinculación son las habilidades necesarias para

\footnotetext{
${ }^{3}$ Véase una explicación más detallada del análisis factorial en la sección III.
} 
el intercambio de información, tecnología y destrezas entre empresas (proveedores, subcontratistas, consultores, instituciones tecnológicas); influyen tanto en la eficiencia productiva de la empresa como en la difusión de la tecnología en la industria, y pueden desarrollarse en distintos niveles. En el nivel primario la empresa adquiere habilidades básicas, en el nivel medio alcanza habilidades secundarias (es decir, de imitación) y en el nivel más elevado logra las habilidades innovativas más altas.

Algunas investigaciones que buscan identificar las fuentes de aprendizaje y las actividades tecnológicas de las empresas se basan en encuestas realizadas especialmente con este objeto, y otras en estadísticas oficiales. En el primer grupo destacan los trabajos siguientes: Westphal, Kritayakirana y otros (1990), realizado con una muestra de 100 empresas tailandesas; Lall y otros (1994), con el caso de cuatro industrias en Ghana; Romijn (1999), centrado en la industria pakistaní de bienes de capital; Wignaraja (2001), con una muestra de empresas textiles y del vestido en las islas Mauricio, y Tremblay (1998), basado en una muestra de la industria papelera canadiense. La complejidad de los aspectos analizados en la construcción del índice es distinta en cada estudio.

Romijn (1999), por ejemplo, mide las capacidades tecnológicas en función del grado de complejidad involucrado en la fabricación de los bienes pertinentes, basando su enfoque en los mecanismos de aprendizaje de la industria metalmecánica a pequeña escala. Señala que la "diversificación hacia arriba" en esta industria ocurre mediante un proceso de aprendizaje por imitación de diseños y reproducción de bienes de fabricación cada vez más compleja, de forma tal que en un mismo parque de maquinaria y equipos es posible fabricar productos distintos, con diferentes grados de complejidad, dependiendo del nivel técnico de las operaciones realizadas; por lo tanto, la manufactura de los distintos productos elaborados con un mismo parque de maquinaria puede requerir diferentes grados de habilidades. Asimismo, en el trabajo citado se desarrolla un indicador de capacidad de producción, más que de innovación o de inversión; se reúne una muestra de 50 productos de la industria local; se diseña un sistema para identificar el nivel de capacidad de producción incorporado en cada bien, y se otorga una calificación al grado de complejidad tecnológica (habilidades y conocimiento) de cada una de las operaciones necesarias para fabricar el producto.

El índice tecnológico de Wignaraja (1998 y 2001) retoma la taxonomía de Lall, pero se estima a partir de dos capacidades tecnológicas, las de producción y las de vinculación, debido a la falta de datos para las de inversión. Examina 40 empresas de la industria del vestido por medio de una encuesta. La categoría de capacidades de producción está representada en el sistema de puntajes por diez actividades técnicas que van desde las tareas comunes de la ingeniería de procesos (tasas de rechazo, estatus según las normas de calidad de los productos industriales — ISO 9000-) a las de ingeniería de producto (copiado, mejora o introducción de nuevos productos). El incremento de la productividad también se incluyó en esta categoría. La categoría de capacidades de vinculación está representada por dos actividades técnicas: la transferencia de tecnología a través de subcontratistas y aquélla por contacto con empresas clientes del extranjero. Cada una de las 12 actividades técnicas que se han mencionado puede ser calificada en distintos niveles, que a su vez reflejan niveles de competencia diferentes en esa capacidad. Así, la posición que ocupe la empresa dependerá del total de puntos que logre de un puntaje máximo de 24 , resultado que se normaliza entre 0 y 1 .

Por último, Tremblay (1998) mide las capacidades tecnológicas ${ }^{4}$ de la industria papelera de Canadá y de India, para examinar la asociación de dichas capacidades con la productividad total de los factores. Cuestiona que en otros estudios no se haya distinguido entre capacidades tecnológicas y de producción, lo que resulta en una visión estrecha de su composición, particularmente en el trabajo de Romijn. Asimismo, Tremblay examina la importancia asignada a las capacidades tecnológicas de los recursos humanos y el descuido de ellas en la estructura de grupos y en la organización en la que los individuos trabajan. Por último, señala que la mayoría de los estudios no incluye las capacidades de generación ni mediciones del cambio técnico. En la investigación de Tremblay los recursos humanos, las habilidades o el capital humano fueron evaluados por aspectos formales, como el número de empleados que se dedican a actividades técnicas y su nivel educativo (en función de tener al menos un grado de licenciatura). Se usaron dos tasas: el número de licenciados como proporción del total de la fuerza de trabajo y el total de licenciados como proporción de las ventas. Utilizando una escala de Likert,

\footnotetext{
${ }^{4}$ En América Latina ha habido intentos de efectuar mediciones de este tipo en Colombia, donde se ha realizado la Encuesta sobre Desarrollo Tecnológico en la Industria Colombiana. Sin embargo, a la fecha de este trabajo no se encontraron resultados en términos de medición de las capacidades tecnológicas.
} 
los esfuerzos de cambio (o el compromiso organizacional con el cambio) se midieron con cuatro variables: escala, intensidad, rol y responsabilidad. La variable escala evaluó la relación entre el número de individuos comprometidos en el cambio y el total de individuos empleados. La variable intensidad evaluó la frecuencia con que se realizaron las actividades de generación de cambio técnico. La variable rol fue definida por el tipo de actividades realizadas (resolución de problemas, ejecución, generación). Finalmente la variable responsabilidad evaluó el compromiso con el cambio de cada miembro de la organización.

Hay otros estudios que utilizan información censal o estadísticas oficiales. Tal es el caso del trabajo de Yan Aw y Batra (1998) sobre la industria taiwanesa. Dichos autores utilizan características específicas de las empresas, como las actividades de investigación y desarrollo y la capacitación en la planta, para cuantificar sus esfuerzos por asimilar la tecnología adquirida. Consideran que también son fuentes de conocimiento otras variables, como la presencia de inversión extranjera directa, el uso de patentes extranjeras y el contacto con clientes internacionales por medio de las exportaciones. Introducen estas características en una función de producción de frontera, para analizar de manera consistente la correlación entre la eficiencia de una empresa y sus inversiones en investigación y desarro1lo, capacitación y vinculación internacional. En el estudio que estamos analizando se reconoce la evidencia ofrecida por Westphal, Rhee y Pursell (1984) sobre la importancia de los clientes extranjeros en la transferencia de tecnología. Yan Aw y Batra suponen que esta adquisición de conocimiento técnico se efectúa a través de las exportaciones. De esta manera, más que estimar un índice compuesto, realizan el análisis en dos pasos. Distinguen dos grupos de empresas, las exportadoras y las que no exportan, y posteriormente estiman funciones de producción de frontera para unas y otras con el fin de evaluar la contribución a la eficiencia de las distintas actividades de aprendizaje.

Por último, Dutrenit y Capdevielle (1993) examinan la evolución de la manufactura, utilizando la clasificación de trayectorias tecnológicas de Pavitt (1984), que incluye una evaluación de las capacidades tecnológicas de las empresas. Para ello utilizan tres variables: las remuneraciones medias (como aproximación a la tecnología de habilidades), la inversión en maquinaria y equipo (como aproximación a la tecnología dura) y la investigación y desarrollo (como aproximación a la tecnología blanda).

Los trabajos que hemos analizado enfrentaron, como bien se señala en ellos, enormes dificultades de información para construir sus índices de capacidades, y se llevaron a cabo en gran medida mediante aproximaciones. Nuestro estudio intenta, en la medida en que se disponga de información, seguir de cerca la taxonomía propuesta por Lall (1992).

Nuestra tarea será simplificar la vasta gama de indicadores de la Encuesta Nacional de Empleo, Salarios, Tecnología y Capacitación (ENESTYC) ${ }^{5}$ para llegar a un número de indicadores más pequeño, pero representativo. Dicha encuesta proporciona un número de variables cuya importancia para la formación de capacidades tecnológicas es incierta. Estimar un índice compuesto otorgando la misma ponderación a todas ellas nos parece incorrecto. Al mismo tiempo, ponderarlas subjetivamente sin tener otra evidencia tampoco parece aceptable. Como se señaló al comienzo, consideramos que la técnica estadística apropiada es en este caso el análisis factorial.

\section{III}

\section{Metodología y fuentes de información}

Toda medida requiere un instrumento y un estándar convenido. La variable producción se mide en toneladas o unidades, la rentabilidad en porcentaje sobre el capital invertido, el costo en unidades monetarias. Las variables que utilizamos en este estudio no se pueden medir de esa manera, ya que por su complejidad conceptual no son observables de manera directa y única. El reto es considerable, sin embargo, es posible aproximarse a las capacidades tecnológicas mediante la construcción de variables que sí se pueden observar de manera directa.

La metodología que se aplica en este estudio corresponde a un análisis multivariado. Las técnicas multivariadas consideradas en un primer momento

\footnotetext{
${ }^{5}$ Que efectúa el Instituto Nacional de Estadística, Geografía e Informática (INEGI) de México.
} 
fueron las de componentes principales o las de análisis factorial. En tanto que las técnicas de componentes principales son más adecuadas para derivar un conjunto pequeño de combinaciones lineales de las variables originales que dé cuenta del total de la varianza, las técnicas de análisis factorial pueden servir mejor para hacer distinciones cualitativas (Schilderinck, 1970; Tabachnick y Fidell, 2001). De ahí que se decidiera utilizar el análisis factorial.

Un factor es una dimensión cualitativa en un eje de coordinadas: define la forma en que las entidades difieren, así como el tamaño de un objeto o su sabor define una dimensión cualitativa. El análisis factorial da una estructura dimensional a los datos, en el sentido de indicar las características comunes presentes en ellos.

En dicho análisis se especifica un modelo formal que describe cada variable mediante unos pocos factores no observables comunes y un factor latente único.

Este último se basa en el supuesto de que hay un número de factores causales que dan lugar a diversas relaciones entre las variables. Otros nombres para estos factores son los de componentes, condicionantes o dimensiones. Su número es considerablemente menor que el número de relaciones. En otras palabras, el análisis factorial descubre dimensiones comunes, o factores que las entrelazan por medio de variables en apariencia no relacionadas; por lo tanto, da luces sobre la estructura subyacente de los datos.

El gran número de variables involucradas en la construcción de los índices de capacidades tecnológicas dificulta el análisis y la deducción de conclusiones para elaborar tales índices. Con el análisis factorial esperamos encontrar un número reducido de variables que expresen los principales elementos condicionantes del aprendizaje en la industria manufacturera mexicana.

Formalmente, el análisis factorial apunta a la selección de un número pequeño de factores comunes que reconstruyen un gran número de variables:

$$
Z_{i j}=\sum_{p=1}^{h} F_{i p} a_{p j}+e_{i j}
$$

donde

$Z=$ las variables observadas

$F=$ los factores comunes no observables directamente de $Z$

$e=$ el factor único análogo al residuo en el análisis de regresión

$a=$ las constantes que se utilizan para combinar los $k$ factores o coeficientes de carga. Estos coeficientes indican el peso que se le asigna a cada factor.
Los factores se pueden inferir de las variables observadas y se pueden estimar como una combinación lineal de ellas de la siguiente manera:

$$
F_{p i}=\sum_{j=1}^{k} q_{p j} z_{j i}
$$

donde

$F=$ el valor del factor

$q=$ el coeficiente de carga

$Z=$ las variables observadas.

La expresión anterior muestra la presencia de patrones de movimiento comunes entre dos o más de las variables en examen. Estos patrones se expresan en coeficientes llamados cargas factoriales que indican en qué medida las varianzas de las variables en cuestión están representadas en este coeficiente por un factor. A su vez, este factor es común a las variables que forman parte de un patrón específico de movimiento (Schilderinck, 1970).

\section{Las fuentes de información}

En este estudio se utilizaron dos fuentes: la ENESTYC y la Encuesta Industrial Anual (EIA). ${ }^{6}$ La ENESTYC contiene información, representativa a escala nacional, sobre las características tecnológicas y de organización productiva, el nivel y tipo de empleo generado, la estructura ocupacional, las remuneraciones y la capacitación de las empresas manufactureras. Su base de datos cubre 8.181 establecimientos. Considera la totalidad de las empresas grandes y medianas, y una muestra aleatoria de las empresas pequeñas y microempresas. Por su parte, la EIA abarca 6.675 establecimientos que cubren 200 clases industriales. Proporciona información estadística sobre el comportamiento de las principales variables económicas del sector manufacturero: entre otras, personal ocupado, remuneraciones, costos y gastos corrientes de operación, energía eléctrica, producción y ventas netas, y activos.

Las variables referentes a la medición de las capacidades tecnológicas se obtuvieron de la ENESTYC de 1999. Esta encuesta consta de 115 preguntas con alrededor de 570 opciones de respuestas. De ellas se seleccionaron inicialmente 50 preguntas con sus opciones de respuestas, que se aproximan a las capacidades identificadas por Lall (1992). Sobre esta base se construyeron 26 variables relacionadas con la inversión, la

\footnotetext{
${ }^{6}$ Como la ENESTYC, la EIA es llevada a cabo por el INEGI.
} 
producción y la vinculación entre empresas e instituciones.

Las variables de aprendizaje e inversión en la empresa son las cinco siguientes: compra de paquetes tecnológicos o transferencia de tecnología desde la empresa matriz, inversiones en tecnología administrativa, inversiones en ingeniería básica, inversiones en patentes y política de reclutamiento de personal.

Para las acciones de cambio en la esfera de la producción se consideraron las siguientes variables: i) investigación y desarrollo, ii) mejoras de la organización, iii) avance en la certificación de la calidad, iv) existencia de mantenimiento preventivo y predictivo, v) presencia de documentación de prácticas y normas, vi) introducción de nuevas tecnologías, vii) asesoría técnica, y viii) intensidad de la capacitación de personal en los cuatro niveles de empleo: personal directivo, empleados, obreros especializados y obreros generales.

Las actividades de vinculación entre empresas se captaron en cinco variables: i) ventas o compras, ii) investigación y desarrollo, iii) capacitación, iv) utilización y adquisición de maquinaria y equipos, y v) acciones de vinculación con universidades $u$ otras instituciones.

Por último, siguiendo el análisis de Yan Aw y Batra (1998) de las capacidades tecnológicas en la industria taiwanesa, se tomó a las exportaciones de la empresa como variable sustitutiva de la información tecnológica proveniente de clientes del exterior. Esto puede aplicarse a cierto tipo de empresas, como las que fabrican partes y componentes, las que utilizan franquicias o las de maquila, ${ }^{7}$ pero no puede hacerse extensivo a todas las empresas que exportan. Consideramos importante incluir esta variable para completar los indicadores sobre las capacidades de vinculación.

Las variables relacionadas con las características de la estructura o dinámica industrial se construyeron con información de la EIA para los años comprendidos entre 1993 y 1998. Estas variables se reflejan en los

\footnotetext{
7 Entre las industrias cuyas exportaciones implican una relación directa con el cliente, que son en gran parte las de maquila, cabe mencionar las que fabrican partes y componentes de vehículos automotores, electrodomésticos y computadores. Sin embargo, en otras industrias es frecuente que, para fines de comercialización, se establezcan alianzas estratégicas con empresas extranjeras que cumplen con la función de transferir conocimientos.
}

indicadores siguientes: la productividad factorial y laboral, el tamaño medio del establecimiento, y la participación de inversión extranjera directa en el capital del establecimiento y en la producción bruta.

Se construyó una muestra con información cruzada de ambas encuestas, identificando en total 1.818 establecimientos. El 73\% son empresas medianas (entre 100 y 500 empleados), de las cuales 265 tienen capital extranjero. Este grupo contribuye con el $34 \%$ del producto bruto, $15 \%$ de las exportaciones y $45 \%$ del personal ocupado de la muestra. Los establecimientos grandes (más de 500 empleados) son 371, de los cuales 115 tienen capital extranjero. Este conjunto aporta el $65 \%$ del producto bruto, $84 \%$ de las exportaciones y $53 \%$ del personal ocupado de la muestra. Como se ve, en la muestra están bien representados los establecimientos medianos y grandes, pero no los de menor tamaño.

Buena parte de las variables son de naturaleza binaria, debido a que así se capturaron en la encuesta. Algunas de ellas abarcaban varios aspectos. Por ejemplo, la variable organización correspondía a la pregunta sobre la posible adopción de cambios en la empresa, como un sistema de producción sincronizado con la demanda ("justo a tiempo"), la rotación de puestos de trabajo, modificaciones en la disposición de las instalaciones y otros. La respuesta positiva en cada uno de estos aspectos valía un punto. Mientras más cambios informaba la empresa, más puntos obtenía con esta variable. Las variables que se capturaron en forma cuantitativa se estimaron como porcentajes y se les asignaron rangos. ${ }^{8}$

Para llegar a los resultados se hicieron varios ejercicios de análisis factorial, que permitieron eliminar aquellas variables con cargas factoriales muy bajas dentro de sus respectivos factores. Las variables eliminadas fueron la inversión en tecnología administrativa, ingeniería básica y asistencia técnica; la inversión en el uso de patentes, y las diversas actividades de vinculación (exceptuando el contacto con clientes extranjeros mediante las exportaciones, la relación con universidades y centros de investigación, la subcontratación y las actividades conjuntas entre empresas).

\footnotetext{
${ }^{8}$ Véase más detalles sobre la construcción de variables en el apéndice A.
} 


\section{IV}

\section{Resultados}

\section{Estimación de los índices de capacidades tec- nológicas}

El resultado del análisis factorial realizado con las variables descritas se presenta en el cuadro 1. En él se identificaron cuatro factores (columnas A, B, C y D) con un valor característico mayor que uno. El método de rotación utilizado fue el de Varimax, que busca minimizar el número de variables que tienen altas cargas en un factor. Las cargas factoriales más altas tienen mayor asociación con la variable correspondiente. Esta matriz factorial permite interpretar las capacidades de la empresa en cuatro dimensiones de aprendizaje que aglutinan a nuestras variables. La columna E del cuadro muestra los coeficientes de comunalidad. ${ }^{9}$

En la columna A del cuadro 1 se aprecian altas cargas factoriales en los porcentajes de personal capacitado en los cuatro niveles de empleo: personal directivo, empleados, obreros especializados y obreros generales. Hay evidencia de que la capacitación de los trabajadores es creciente, tanto en horas impartidas como en calidad, aunque sin duda no está generalizada. En nuestra opinión, esta pauta de comportamiento de las empresas pone de manifiesto la presencia en ellas de una política de formación de personal de carácter integral. De ahí el encabezado de dicha columna.

En la columna B se observa un interesante patrón de relaciones entre variables: avance en los sistemas de calidad y la certificación de la calidad, cambios en la organización del proceso productivo, adquisición de paquetes tecnológicos y transferencia de tecnología, políticas de reclutamiento de personal, de investigación y desarrollo y de aprendizaje por el contacto con clientes internacionales a partir de las exportaciones. Las cargas factoriales más altas corresponden a compra de tecnología $(0,47)$, cambios en los sistemas de calidad y certificación de la calidad $(0,46)$, cambios en la organización del proceso de trabajo,

\footnotetext{
${ }^{9}$ Esta información es fundamental en el análisis factorial; no así en el método de componentes principales, en que se da cuenta del porcentaje de la varianza explicada. Como señalan Dillon y Golstein (1984), el análisis de componentes principales es un análisis de varianza, en tanto que el análisis factorial centra su atención en la cantidad de varianza que cada variable comparte con las otras. Por lo tanto, el análisis factorial es un análisis de covarianza.
}

aprendizaje por el contacto con clientes extranjeros a partir de las exportaciones $(0,33)$ e investigación y desarrollo en la empresa $(0,31)$.

Como ocurre con otros países de industrialización tardía, México depende de la adquisición de tecnología del extranjero. Esta variable aparece interrelacionada con otras variables de aprendizaje que son parte de lo que puede denominarse innovación de mejora continua. Las innovaciones en los procesos productivos han significado en las empresas una búsqueda intensa de la resolución de problemas. Las actividades consiguientes han derivado en cambios en la organización de las operaciones productivas que pueden involucrar una nueva disposición de las instalaciones, la adopción de sistemas de producción justo a tiempo, la formación de círculos de calidad y una mayor participación de los trabajadores (Coriat, 1992). Asimismo, las empresas avanzan paulatinamente hacia lo que se denomina una nueva cultura de la calidad. Esto implica adoptar un enfoque sistémico de medición a fin de dar un mejor servicio al cliente, pero también significa rehacer menos trabajos y bajar costos, lo que ha sido muy evidente en la industria automotriz (Carrillo, 1993). Los departamentos de producción y calidad dejan de hallarse separados y pasan a estar en continua comunicación. La presencia de la variable de investigación y desarrollo en la empresa denota su conexión con los esfuerzos de asimilación, adaptación y mejora de la tecnología importada, como ocurre en países similares, y no una etapa de las capacidades de innovación. Por último, el aprendizaje por el contacto con clientes extranjeros a partir de las exportaciones puede sugerir que el flujo de información se relaciona con este proceso de innovación de mejora continua, el que a su vez permite mayores exportaciones. La variable referida a la política de reclutamiento de la empresa denota ante todo la calificación necesaria del empresario para emprender estos cambios.

En la columna $\mathrm{C}$, las cargas factoriales de las variables relativas a la documentación de planes de capacitación y de programas de seguridad ponen de relieve la importancia de desarrollar sistemas de información y documentación en las empresas. La selección de indicadores precisos permite efectuar un análisis certero encaminado a la identificación de problemas 
México: Resultados del análisis factorial, 1999a b

\begin{tabular}{|c|c|c|c|c|c|}
\hline & \multicolumn{4}{|c|}{ Factores } & \multirow{2}{*}{$\begin{array}{c}\text { Coeficientes } \\
\text { de } \\
\text { comunalidad }^{\mathrm{c}}\end{array}$} \\
\hline & $\begin{array}{l}\text { Política de } \\
\text { formación } \\
\text { de personal } \\
\text { (A) }\end{array}$ & $\begin{array}{l}\text { Innovación } \\
\text { en mejora } \\
\text { continua } \\
\text { (B) }\end{array}$ & $\begin{array}{c}\text { Sistemas de } \\
\text { información y } \\
\text { documentación } \\
\text { (C) }\end{array}$ & $\begin{array}{l}\text { Inversión } \\
\text { en nuevas } \\
\text { tecnologías } \\
\text { (D) }\end{array}$ & \\
\hline Cambios en la organización & 0,01 & $\mathbf{0 , 4 0}$ & 0,16 & 0,11 & 0,20 \\
\hline Cambios en los sistemas y certificación de calidad & 0,05 & 0,46 & 0,12 & 0,07 & 0,23 \\
\hline Política de reclutamiento de personal & 0,13 & $\mathbf{0 , 2 8}$ & 0,12 & 0,15 & 0,13 \\
\hline Documentación de programas de capacitación & 0,16 & 0,17 & $\mathbf{0 , 5 9}$ & 0,11 & 0,41 \\
\hline Documentación de programas de seguridad y normas & 0,04 & 0,16 & $\mathbf{0 , 5 9}$ & $-0,02$ & 0,38 \\
\hline$\%$ de pers. directivo capacitado & 0,49 & 0,19 & 0,09 & 0,02 & 0,29 \\
\hline$\%$ de empleados capacitados & $\mathbf{0 , 8 3}$ & 0,06 & 0,04 & 0,02 & 0,70 \\
\hline$\%$ de obreros especializados & 0,67 & 0,07 & 0,06 & 0,05 & 0,46 \\
\hline$\%$ de obreros generales capacitados & 0,66 & $-0,08$ & 0,05 & $-0,04$ & 0,45 \\
\hline las exportaciones & 0,04 & $\mathbf{0 , 3 3}$ & 0,01 & 0,09 & 0,12 \\
\hline Introducción de tecnología CNC y robots & $-0,01$ & 0,15 & 0,04 & 0,65 & 0,45 \\
\hline Renovación de equipos y nuevas tecnologías & 0,01 & 0,172 & 0,02 & 0,272 & 0,10 \\
\hline Investigación y desarrollo & 0,08 & $\mathbf{0 , 3 1}$ & 0,06 & 0,15 & 0,13 \\
\hline
\end{tabular}

Fuente: Estimaciones propias con información de la Encuesta Nacional de Empleo, Salarios, Tecnología y Capacitación (ENESTYC) de 1999.

a Método de extracción: factor de eje principal (principal axis factoring). Rotación de Varimax con normalización de Kaiser. La rotación convergió en cinco iteraciones.

b Las negritas destacan las cargas factoriales altas.

c Los coeficientes de comunalidad miden la relación entre la varianza de cada variable y la varianza del resto de las variables.

críticos, gracias a lo cual las empresas pueden emprender la resolución de estos problemas con una visión de largo plazo, y también hacer el seguimiento de los resultados. Sin embargo, estas variables son sólo una aproximación a lo que denominamos sistemas de información y documentación. La documentación de los programas de capacitación involucra un conjunto de temas que van desde los contenidos hasta las formas de evaluación y seguimiento. Algo similar sucede con la documentación de los programas de seguridad. Por lo general las empresas que emprenden este tipo de tareas ya cuentan con otros niveles de documentación más básicos, como el de las normas y procedimientos formales.

En la columna D se aprecia el esfuerzo tecnológico desde la perspectiva de la utilización de tecnología dura: la introducción de nuevas tecnologías y la renovación de equipos. Parte del conocimiento está, sin duda, incorporado a las máquinas. En la medida en que éstas se renuevan, la empresa adquiere nuevos conocimientos y se ve alentada a realizar cambios complementarios. Distintos estudiosos han analizado la fuerte relación recíproca que existe entre la aplicación de nuevas tecnologías y los cambios organizacionales (Edquist, 1992; Hoffman, 1989). Un cambio en la or- ganización puede traer consigo un cambio tecnológico, o viceversa, o bien ambos pueden acompañarse y condicionarse mutuamente.

La columna E presenta los coeficientes de comunalidad, ${ }^{10}$ que son relativamente satisfactorios. Se observa que estos son más altos en las actividades de capacitación y documentación. Los coeficientes más bajos (inferiores a 0,20) corresponden a las variables renovación de equipo, investigación y desarrollo, y política de reclutamiento de personal. Aun así, se decidió no eliminar estas variables en el análisis porque son complementarias de los factores identificados.

En suma, la utilización del análisis factorial permitió distinguir las cuatro dimensiones más representativas del aprendizaje tecnológico en las empresas industriales mexicanas. Se identificaron de preferencia variables relativas a las capacidades de producción identificadas en la taxonomía de Lall. De las variables de inversión solamente aparecen la compra de tecnología o de paquetes tecnológicos y la política de reclutamiento de personal altamente calificado.

\footnotetext{
${ }^{10}$ Como se dijo en el cuadro 1 , estos coeficientes miden la relación entre la varianza de cada variable y la varianza del resto de las variables.
} 
Llama la atención que, en la variable de vinculación, la relación con universidades y centros de investigación, la subcontratación y las actividades conjuntas entre empresas hayan quedado relegadas en el análisis factorial a los últimos lugares, con un coeficiente de comunalidad inferior a 0,10 . Una excepción notable, y que se consideró separadamente de dicha variable, fue el contacto con clientes extranjeros a través de las ventas al exterior. Indudablemente, ha habido avances en las actividades de vinculación, presentes en algunas de las empresas más destacadas, pero en una muestra de gran tamaño no resultan significativas. En otras palabras, se confirman resultados obtenidos por otros autores en cuanto a que todavía es insuficiente el contacto de las empresas mexicanas entre ellas y con las universidades (Casas, de Gortari y Luna, 2000).

\section{Análisis de agrupamientos}

A partir de la información obtenida del análisis factorial se calculó el puntaje de cada factor en cada una de las observaciones. Los puntos factoriales representan la relación de las distintas observaciones con cada factor, y son altos cuando los coeficientes de comunalidad y la razón entre las variables y los factores son elevados.

El análisis de agrupamientos de empresas se llevó a cabo utilizando los puntos factoriales, mediante el método conocido como $K$ means. Éste consiste en identificar grupos de casos relativamente homogéneos y se basa en un algoritmo que minimiza la distancia euclidiana entre el caso $i$ y el promedio del grupo que contiene este caso. El procedimiento consiste en mover $\operatorname{los} n$ casos de un grupo a otro hasta llegar al punto en que ninguno de estos traslados disminuye el error en la partición, siendo este error la sumatoria al cuadrado de las distancias euclidianas.
$\mathrm{El}$ análisis de agrupamientos que se presenta en el cuadro 2 identifica varios patrones distintos de acumulación de capacidades entre las empresas de la muestra, con base en los cuatro factores mencionados anteriormente. El perfil de los cuatro grupos identificados se resume como sigue. El grupo I consta de 741 establecimientos con el $62 \%$ del producto bruto y $75 \%$ de las exportaciones totales de la muestra. Tiene el nivel más alto de capacidades de innovación mediante la mejora continua y la inversión en nuevas tecnologías, y el segundo más alto en sistemas de información y documentación. Está compuesto primordialmente por empresas grandes, que generan el $73 \%$ del producto bruto del grupo, e incluye 204 empresas con capital extranjero, que originan el $52 \%$ del producto bruto y concentran el $78 \%$ de las exportaciones de la muestra. El tamaño medio de los establecimientos que lo integran, medido por la producción bruta y las exportaciones, es mayor que en los otros tres grupos (cuadros 2 y 3 ).

El grupo II está integrado por 169 empresas que generan el 10\% del valor bruto de la producción y de las exportaciones de la muestra. Tiene el nivel más alto en política de formación de personal y un nivel medio en sistemas de información y documentación. Al igual que en el primer grupo, prevalecen en él empresas grandes que originan el $70 \%$ del producto bruto y $90 \%$ de las exportaciones de la muestra. Sólo incluye 29 empresas con participación extranjera. El tamaño medio de los establecimientos, medido por el producto bruto y por la exportaciones, respectivamente, es de $30 \%$ y $40 \%$, es decir, menor que el del primer grupo (cuadros 3 y 4 ).

El grupo III tiene el nivel más alto en sistemas de información y documentación y no presenta ninguna diferencia en otros aspectos. Está compuesto por 627 empresas que generan $24 \%$ del valor bruto de la producción y $13 \%$ de las exportaciones de la muestra.

CUADRO 2

México: Características de grupos de empresas, 1999

\begin{tabular}{|c|c|c|c|c|c|}
\hline \multirow[t]{2}{*}{ Grupos } & \multirow[t]{2}{*}{ Núm. } & \multicolumn{2}{|c|}{$\begin{array}{l}\text { Tamaño de la empresa media } \\
\text { (en miles de pesos de 1993) }\end{array}$} & \multirow{2}{*}{$\begin{array}{c}\text { Establecimientos con } \\
\text { participación de IED } \\
\text { (número) }\end{array}$} & \multirow{2}{*}{$\begin{array}{l}\text { Participación en el } \\
\text { producto bruto de los } \\
\text { establecimientos } \\
\text { con IED }(\%)\end{array}$} \\
\hline & & Producto bruto & Exportaciones & & \\
\hline I & 741 & 272154 & 63873 & 204 & 52 \\
\hline II & 169 & 193620 & 38060 & 29 & 32 \\
\hline III & 627 & 124717 & 13577 & 141 & 37 \\
\hline IV & 247 & 61769 & 3948 & 24 & 9 \\
\hline
\end{tabular}

Fuente: Estimaciones propias con información de la Encuesta Industrial Anual (EIA). 


\begin{tabular}{lcccc}
\hline Grupos & Número & $\begin{array}{c}\text { Política de formación } \\
\text { de personal }\end{array}$ & $\begin{array}{c}\text { Innovación de } \\
\text { mejora continua }\end{array}$ & $\begin{array}{c}\text { Sistemas de información } \\
\text { documentación }\end{array}$ \\
\hline I & 741 & $-0,187$ & 0,2753 & 0,2515 \\
II & 169 & 2,045 & 0,0087 & 0,1674 \\
III & 627 & $-0,1799$ & $-0,0801$ & 0,335 \\
IV & 247 & $-0,5312$ & $-0,4853$ & $-1,4933$ \\
\hline
\end{tabular}

Fuente: Estimaciones propias con información de la Encuesta Nacional de Empleo, Salarios, Tecnología y Capacitación (ENESTYC)

CUADRO 4

México: Características de los grupos de empresas, 1993-1998

\begin{tabular}{|c|c|c|c|c|c|}
\hline Grupos & $\begin{array}{l}\text { Margen de } \\
\text { ganancia }\end{array}$ & $\begin{array}{l}\text { Productividad } \\
\text { laboral }\end{array}$ & $\begin{array}{l}\text { Productividad total } \\
\text { de los factores }\end{array}$ & Cambio técnico & Eficiencia \\
\hline I & 1,150 & 1,326 & 1,008 & 1,010 & 1,017 \\
\hline II & 1,025 & 1,036 & 0,999 & 1,031 & 1,006 \\
\hline III & 0,958 & 0,943 & 1,006 & 1,002 & 0,992 \\
\hline IV & 0,867 & 0,696 & 0,987 & 0,957 & 0,985 \\
\hline Promedio & 1,000 & 1,000 & 1,000 & 1,000 & 1,000 \\
\hline \multicolumn{6}{|c|}{ Análisis de la varianza } \\
\hline $\mathrm{F}$ & 9,24 & 15,63 & 1,20 & 4,77 & 0,61 \\
\hline Probabilidad & 0,00 & 0,00 & 0,31 & 0,00 & 0,61 \\
\hline
\end{tabular}

Fuente: Estimaciones propias con información de la Encuesta Industrial Anual (EIA), varios años.

Hay 141 establecimientos con participación extranjera, los que aportan el $37 \%$ del producto bruto y $51 \%$ de las exportaciones de dicha muestra (cuadros 3 y 4).

El grupo IV incluye 247 empresas que aportan el $5 \%$ del producto bruto y sólo el $2 \%$ de las exportaciones de la muestra. Tiene los niveles más bajos en todos los factores. El tamaño de la empresa media es más de cuatro veces menor que en el grupo I y bastante inferior al de los grupos II y III. Debe recordarse que la muestra analizada aquí corresponde a 1.881 empresas de las 8.181 que abarca la ENESTYC, dado que no se contaba con información sobre el desempeño en el tiempo de todas ellas. Dicha muestra está ciertamente sesgada hacia las mejores empresas. Haciendo un ejercicio similar para el conjunto de los establecimientos considerados en la ENESTYC, se verificó que el grupo I incluía 1.981 empresas; el II, 869; el III, 2.014, y el IV, 3.357. Así, si se considera el total de la ENESTYC, el grupo con menores capacidades abarca el $41 \%$ del total de los establecimientos y el que le sigue hacia arriba el $24 \%$.

Hubiera sido de esperar que en un grupo, particularmente el grupo I, se encontrara que todos los factores eran positivos. El que no ocurriera así en el aná- lisis de agrupamientos no significa que no existan empresas que tengan esas características. Encontramos que en 76 de las 1.818 empresas los cuatro factores aparecían positivos, y que lo mismo sucedía en 696 de los 8.181 establecimientos incluidos en la ENESTYC (la mayor parte de esos 696 correspondía a los grupos I y II). Por otra parte, el hecho de que el grupo I no haya destacado en política de formación integral de personal no significa que estos establecimientos le den poca importancia a la capacitación.

\section{3. ¿Están asociados los agrupamientos con los niveles de desempeño?}

Para responder a esta pregunta se compara el desempeño de los grupos considerados en términos de margen bruto de ganancia, productividad laboral, y productividad factorial estimada con el índice de Malmquist, que permite descomponerla en cambio técnico y eficiencia. ${ }^{11}$ Como señalan Cantner y Hanusch (2001), el

\footnotetext{
11 Véase en el apéndice B más detalles acerca del uso de la metodología de Malmquist para estimar los índices de productividad total de los factores, el cambio técnico y la eficiencia.
} 
uso de la productividad factorial puede parecer algo pasado de moda, dadas las críticas surgidas principalmente en el contexto de ejercicios de contabilidad del crecimiento, donde la determinación de la productividad factorial se basa en supuestos de equilibrio y condiciones de la teoría tradicional, combinada con el supuesto de que la misma función de producción es aplicada a todas las observaciones. El índice de Malmquist no tiene estos supuestos y además permite identificar tanto el cambio técnico local en la frontera productiva como las observaciones que están por debajo de la mejor práctica. ${ }^{12}$ El grupo I, que destaca por sus altos índices de capacidades tecnológicas, presenta los mayores índices de desempeño, salvo en materia de cambio técnico, donde el grupo II es ligeramente superior (1.031 frente a 1.010) ${ }^{13}$ Es decir, las empresas con prácticas de documentación y planeación, renovación de equipos y maquinaria y programas com-

\section{V}

\section{Conclusiones}

Este trabajo tiene el propósito de hacer un aporte metodológico y analítico a la línea de investigaciones abocadas a construir índices representativos de las capacidades tecnológicas de las empresas manufactureras mexicanas, y se ha preocupado de examinar la distribución de estas capacidades en una muestra de empresas.

En lo que se refiere a la construcción de índices representativos de las capacidades tecnológicas, el empleo del análisis factorial nos permitió simplificar relaciones complejas en un número reducido de factores (o dimensiones) comunes que las entrelazan mediante variables aparentemente no relacionadas. La ventaja de esta aproximación frente a la de computar un índice único mediante puntajes simples, como han hecho otros autores, es que evitamos dar ponderaciones subjetivas y dejamos al análisis factorial la tarea de determinar el porcentaje de la varianza que es explicada por cada factor. Esta es una contribución del presente trabajo.

12 Véase en Cantner y Hanusch (2001) un completo análisis de la utilización del índice de Malmquist en un contexto de heterogeneidad y cambio evolutivo.

${ }^{13}$ Los índices se normalizaron respecto a los promedios de la muestra. plejos de mejora continua tienen mejor desempeño. Algo similar ocurre con el grupo II, que tiene mejor desempeño que los siguientes, con una excepción. Esto sugiere que hay una asociación positiva entre capacidades tecnológicas y desempeño.

Con el fin de determinar si las diferencias son significativas se realizó un análisis de la varianza en un sentido, cuyos resultados se muestran en los dos últimos renglones del cuadro 5. Las diferencias observadas son significativas en tres de los cinco índices: el margen de ganancia, la productividad laboral y el cambio técnico, lo cual respalda la hipótesis de que las capacidades tecnológicas son un elemento decisivo en la innovación y el desempeño empresarial. Un análisis de la varianza entre cada grupo y el resto de ellos (prueba de Scheffe) permite verificar que las mayores diferencias ocurren entre el grupo I y el grupo IV, el rezagado. ${ }^{14}$

En el caso que nos ocupa, después de un exhaustivo análisis factorial exploratorio fue posible identificar cuatro factores que expresan las principales fuentes de aprendizaje en la empresa manufacturera: i) la política de formación de personal, ii) la innovación de mejora continua, iii) los sistemas de información y documentación, y iv) la inversión en nuevas tecnologías.

El primer factor, como su nombre lo indica, expresa la definición de una política de formación del personal en todos los niveles de la empresa: personal directivo, empleados, obreros especializados y obreros generales. Se observa un alto grado de correlación entre estas variables, lo cual sugiere que cuando una empresa tiene una política de capacitación, la tiene a todos los niveles.

El segundo factor está ligado a las actividades de aprendizaje mediante la mejora continua. La presencia de la labor de investigación y desarrollo en este grupo de variables sugiere que, en la industria mexicana, esta labor está vinculada con los esfuerzos de asimilación, adaptación y mejora de tecnología importada - como ocurre en países similares - y no corresponde a una etapa de capacidades innovativas. Las

\footnotetext{
${ }^{14}$ Véase en el apéndice C el resultado de la prueba de Scheffe.
} 
actividades de aprendizaje están relacionadas con la actividad exportadora, nuestra variable aproximativa a la información tecnológica proveniente de clientes del exterior, como proponen Yan Aw y Batra (1998) en el caso de la industria taiwanesa. El proceso de modernización en México ha significado para las empresas una búsqueda intensa de resolución de problemas, más que de innovaciones radicales, lo que ha derivado en cambios en la organización de las operaciones productivas. Asimismo, las empresas avanzan paulatinamente hacia lo que se denomina una nueva cultura de la calidad, con certificaciones de calidad internacionales. Por último, el aprendizaje por medio del contacto con clientes extranjeros a partir de las exportaciones puede sugerir que el flujo de información logrado tiene vínculos con el proceso de innovación de mejora continua, que a su vez permite acrecentar las exportaciones

El tercer factor identifica los sistemas de documentación de procesos, normas escritas y planeación de la empresa. No es posible adoptar un enfoque de largo plazo sin una selección de indicadores precisos que permitan efectuar análisis certeros para identificar problemas críticos.

El cuarto factor, por último, expresa el esfuerzo tecnológico desde la perspectiva de la utilización de tecnología dura, es decir, la introducción de nuevas tecnologías y la renovación de maquinaria.

Aunque nuestras variables iniciales fueron seleccionadas siguiendo la taxonomía de Lall (1992), al final del análisis las que predominaron fueron las capacidades de producción. En lo que toca a las capacidades de inversión, es preciso mencionar la compra de tecnología, las actividades de investigación y desarrollo, y la renovación de equipo e introducción de nuevas tecnologías.

Los resultados de la variable vinculación, en lo que se refiere a la relación con universidades y centros de investigación, la subcontratación y las actividades conjuntas entre empresas, expresan sin duda una de las insuficiencias mayores en la capacidad de innovación de las empresas mexicanas. Hay avances debidos en menor medida al acercamiento de algunas empresas a universidades, que no se reflejan a nivel de la muestra, pero en medida mayor al contacto con clientes extranjeros por medio de las exportaciones.

Para examinar la distribución de las capacidades en la muestra utilizamos el análisis de agrupamientos, tomando como base el puntaje de cada observación para cada factor. Se identificaron cuatro grupos de empresas en función de sus índices factoriales de capacidades. El grupo I presenta tres factores con niveles positivos: sistemas de documentación y planeación, introducción de nuevas tecnologías e innovación de mejora continua. El grupo II exhibe intensos programas de capacitación y sistemas de planeación y documentación, seguido por el grupo III, que sólo tiene sistemas de documentación y planeación. El grupo IV acusa niveles negativos en los tres factores, o sea, es el más atrasado.

Si bien cabía esperar que la capacitación estuviera presente en el grupo I, las empresas de este grupo tienen un promedio bajo en capacitación; en nuestra opinión, es posible que la hayan realizado en el pasado y que esto no se haya reflejado en el momento de la entrevista; pero en todo caso, en relación con el segundo factor, correspondiente a la innovación de mejora continua, se aprecia que las empresas tienen políticas de reclutamiento de personal altamente calificado. Como ya se explicó, el bajo número de establecimientos en el grupo IV no indica que esto sea representativo de la industria, ya que la muestra está sesgada hacia establecimientos medianos y grandes. En una muestra mayor, el grupo más rezagado incluye el $41 \%$ de los establecimientos. La evidencia sobre el atraso estructural de las microempresas y pequeñas empresas sugiere que el porcentaje mayor de establecimientos en la industria mexicana corresponde a este grupo.

Las características de los grupos confirman la asociación entre el tamaño de las empresas y el nivel de sus capacidades tecnológicas. Nuestros resultados matizan los efectos de la inversión extranjera directa en dichas capacidades. Es claro que hay un número importante de empresas extranjeras en el grupo que exhibe el nivel más alto de capacidades tecnológicas. Pero no todas las empresas extranjeras tienen estas características, como lo muestra el elevado porcentaje de ellas que se encuentra en el grupo III.

Por último, es necesario volver al planteamiento inicial respecto a la importancia de analizar el nivel de las capacidades tecnológicas de las diversas empresas, para entender mejor sus diferencias en la situación de heterogeneidad que caracteriza a las economías en desarrollo. El análisis de los indicadores de desempeño en los grupos con distintos niveles de capacidades tecnológicas mostró una asociación de las capacidades tecnológicas y el desempeño en el caso de la productividad laboral, el margen de ganancia y el cambio técnico. Esto indica que las capacidades tecnológicas, a través de su efecto en el comportamiento innovativo de las empresas, influyen positivamente en el desempeño y contribuyen a explicar las diferencias que se observan en éste. 
Dado que los promedios de desempeño son decrecientes desde el grupo I al IV, resulta tentador sugerir que la distribución de los grupos según los puntos factoriales podría reflejar un patrón de la acumulación de capacidades en las empresas. La adquisición de capacidades tecnológicas a partir del desarrollo de sistemas de documentación y planeación (grupo III) sería un mínimo necesario para el aprendizaje; luego, en orden ascendente, vendrían los programas de capacitación en la planta (grupo II), y en seguida el esfuerzo más completo de aprendizaje por medio de la innovación de mejora continua (grupo I), que en el caso mexicano incluye investigación y desarrollo. En esto se corre el riesgo de presentar un análisis demasiado simplista. La prueba de Scheffe sólo avaló este posible patrón de acumulación de capacidades en el caso de la productividad del trabajo y del margen de ganancias, donde las diferencias son significativas entre el grupo I y los grupos restantes. En el caso del cambio técnico, las grandes diferencias se dan entre el grupo IV (el rezagado) y el resto. De ahí que haya margen para la selección de estrategias empresariales de aprendizaje derivadas de las necesidades sectoriales de las empresas y no necesariamente un avance en etapas definidas. Sin embargo, ésta es una línea de investigación que está pendiente y en la cual hay que avanzar. Si se demostrara, por ejemplo, que la documentación es un primer paso, esto tal vez llevaría a que la política tecnológica e industrial estableciera prioridades al respecto.

En todo caso, nuestros resultados apuntan a la necesidad de delinear una política tecnológica para apoyar la acumulación de capacidades tecnológicas empresariales. A la fecha ha habido algunos progresos en los programas de apoyo, pero su cobertura sigue siendo insuficiente. Por lo demás, hemos encontrado que son pocas las empresas que tienen una estrategia integral de aprendizaje tecnológico. 
APENDICE A

Metodología para construir las variables a partir de la Encuesta Nacional de Empleo, Salarios, Tecnología y Capacitación (ENESTYC)

\begin{tabular}{|c|c|c|c|}
\hline Grupo & Variables & $\begin{array}{l}\text { Unidad en que se } \\
\text { expresa en la encuesta }\end{array}$ & $\begin{array}{l}\text { Criterio para asignar } \\
\text { valores a la variable }\end{array}$ \\
\hline $\begin{array}{l}\text { Aprendizaje e } \\
\text { inversión }\end{array}$ & $\begin{array}{l}\text { Compra de paquetes tecnológicos o } \\
\text { transferencia de la empresa matriz } \\
\text { Inversiones en tecnología administrativa } \\
\text { Inversiones en ingeniería básica } \\
\text { Inversiones en compra de patentes } \\
\text { Política de reclutamiento de personal calificado } \\
\text { para cada nivel de empleo }\end{array}$ & $\begin{array}{l}\text { \% sobre la inversión } \\
\text { “" } \\
\text { "“ } \\
\text { Número de empleados } \\
\text { por nivel de educación } \\
\text { formal requerido para } \\
\text { personal directivo, } \\
\text { empleados y obreros }\end{array}$ & $\begin{array}{l}\text { Rangos de } 1 \text { a } 3 \\
\qquad \text { “" } \\
\text { Si directivos deben tener } \\
\text { al menos bachillerato } \\
\text { W=1 } \\
\text { Si empleados deben } \\
\text { tener al menos } \\
\text { bachillerato, } \\
\mathrm{x}=1 \\
\text { Si los obreros (generales } \\
\text { o especializados) deben } \\
\text { tener secundaria } \\
\text { completa } \\
\text { y= } 1 \text { y } \mathrm{z}=1 \\
\text { Política de reclutamiento } \\
=\mathrm{w}+\mathrm{x}+\mathrm{y}+\mathrm{z} \\
\text { Valor máximo }=4\end{array}$ \\
\hline Producción & $\begin{array}{l}\text { Investigación y desarrollo aplicados al proceso } \\
\text { productivo } \\
\text { Organización } \\
\text { (producción justo a tiempo+cepp }{ }^{\text {a }}+\text { rotación } \\
\text { +disposición de las instalaciones +participación } \\
\text { de los trabajadores +supervisión+estándares) } \\
\text { Calidad } \\
\text { (círculos de calidad +control total de } \\
\text { calidad+certificación +c. instrumental) } \\
\text { Enfoque de mantenimiento preventivo y } \\
\text { predictivo } \\
\text { Documentación (normas + capacitación) } \\
\text { Seguridad } \\
\text { Introducción de nuevas tecnologías: Compra } \\
\text { de tecnología } \\
\text { Intensidad de capacitación de personal } \\
\text { (personal directivo,empleados, obreros } \\
\text { especializados, obreros generales) }\end{array}$ & $\begin{array}{l}1=\text { sí } \\
1=\text { sí } \\
1=\text { sí } \\
1=\text { sí } \\
1=\text { sí } \\
1=\text { sí } \\
\text { CNC o robots=1 } \\
1=\text { sí } \\
\text { Número de trabajadores } \\
\text { o empleados capacitados }\end{array}$ & $\begin{array}{l}1,0 \\
\text { Valor máximo= } 7 \\
\text { Valor máximo= } 4 \\
1,0 \\
1,0 \\
1,0 \\
1,0 \\
\begin{array}{l}1=\text { de } 1 \% \text { a } 20 \% \\
2=\text { de } 20 \text { a } 39 \% \\
3=\text { más de } 40 \%\end{array}\end{array}$ \\
\hline Vinculación & $\begin{array}{l}\text { Subcontratación } \\
\text { Vinculación con universidades } \\
\text { Actividades conjuntas en: } \\
\text { Ventas o compras } \\
\text { Investigación y desarrollo } \\
\text { Capacitación } \\
\text { Utilización y compra de maquinaria y equipo } \\
\text { Acciones de vinculación con universidades u } \\
\text { otras instituciones } \\
\text { Flujos de información por contactos con } \\
\text { clientes del extranjero }\end{array}$ & $\begin{array}{l}1=\text { sí } \\
1=\text { sí } \\
1=\text { sí } \\
1=\text { sí } \\
1=\text { sí } \\
1=\text { sí } \\
1=s i ́ \\
1=\text { sí }\end{array}$ & De 1 a 8 \\
\hline
\end{tabular}

Fuente: elaboración propia.

a Control del estado de la producción y el proceso. 
La construcción de este índice está basada en el concepto de las funciones distancia del producto. ${ }^{15}$ Para cada uno de los períodos estudiados, la tecnología $S^{16}$ es el conjunto de insumos $\left(x^{t}=\left(x^{l}, \ldots \ldots \ldots, x^{N}\right)\right)$ y productos $\left(y^{t}=\left(y^{l}, \ldots \ldots \ldots, y^{N}\right)\right)$. La función distancia del producto en el periodo $t$, expresada como $D_{0}^{t}\left(x^{t}, y^{t}\right)^{17}$ mide la máxima expansión de la producción que es posible alcanzar con un vector dado de insumos ${ }^{18}$ respecto a una función frontera. La función distancia del producto para una industria dada tiene el valor de uno cuando el nivel del producto corresponde al de la frontera de producción; es menor que uno cuando el nivel de la producción está por debajo del de frontera, y es mayor que uno cuando el nivel de la producción está por encima del de frontera.

De manera similar, la función distancia $D_{1}^{t+1}\left(x^{t}, y^{t}\right)$ es la relación entre el producto alcanzado en el periodo $t$ y el máximo posible dada la tecnología del periodo $t+1$.

Según Caves, Christensen y Diewert (1982), el índice Malmquist de productividad total de los factores entre dos períodos, por ejemplo $t$ y $t+1$, puede tener como punto de referencia la tecnología de cualquiera de los dos años. Tomando como parámetro la tecnología del año $t$, el índice es el siguiente $M_{i}^{t}=\frac{D_{0}^{t}\left(x^{t+1}, y^{t+1}\right)}{D_{0}^{t}\left(x^{t}, y^{t}\right)}$ Cuando se toma como parámetro la tecnología del periodo $t+1$ el índice se define de la siguiente manera: $M_{1}^{t+1}=\frac{D_{0}^{t+1}\left(x^{t+1}, y^{t+1}\right)}{D_{0}^{t+1}\left(x^{t}, y^{t}\right)}$. Cuando $M>1$, denota un aumento de la productividad entre el período $t$ y $t+1$ y cuando $\mathrm{M}<1$ denota una disminución de la productividad.
Färe, Grosskopf y Zhang (1994) demostraron que puede considerarse el índice de Malmquist como una media geométrica de las dos anteriores ecuaciones.

$$
M_{i}\left(y^{t+1}, x^{t+1}, y^{t}, x^{t}\right)=\left[\frac{D_{0}^{t}\left(y^{t+1}, x^{t+1}\right)}{D_{0}^{t}\left(y^{t}, x^{t}\right)}\right]\left[\frac{D_{0}^{t+1}\left(y^{t+1}, x^{t+1}\right)}{D_{0}^{t+1}\left(y^{t}, x^{t}\right)}\right]^{1 / 2}
$$

El índice así definido puede descomponerse en dos partes: cambio en la eficiencia (acercarse a la frontera) y cambio técnico (innovación). De la siguiente manera:

$M_{0}\left(y^{t+1}, x^{t+1}, y^{t}, x^{t}\right)=\left[\frac{D_{0}^{t+1}\left(y^{t+1}, x^{t+1}\right)}{D_{0}^{t}\left(y^{t}, x^{t}\right)} x\right]\left[\left(\frac{D_{0}^{t}\left(y^{t+1}, x^{t+1}\right)}{D_{0}^{t+1}\left(y^{t+1}, x^{t+1}\right)}\right)\left(\frac{D_{0}^{t}\left(y^{t}, x^{t}\right)}{D_{0}^{t+1}\left(y^{t}, x^{t}\right)}\right]^{1 / 2}\right.$

El método considera la posibilidad de trabajar con rendimientos constantes a escala y variables. Los rendimientos constantes a escala son apropiados cuando se supone que todas las empresas producen a una escala óptima. Sin embargo, la competencia imperfecta y otros obstáculos, como pueden ser los financieros, ocasionan que las empresas no se encuentren produciendo a esa escala óptima. Cuando las empresas no tienen escalas óptimas y se suponen rendimientos constantes a escala el cambio técnico se confunde con eficiencias en la escala. Para separar del cambio técnico el efecto de la escala es necesario calcular el índice del cambio técnico en conformidad con el supuesto de rendimientos constantes y variables a escala. La diferencia entre estos dos índices indica una eficiencia o ineficiencia en la escala.

APENDICE C

Análisis de la varianza en un sentido: prueba de Scheffe

\begin{tabular}{|c|c|c|c|c|c|c|}
\hline Grupo & Grupo & $\begin{array}{l}\text { Productividad } \\
\text { total de los factores }\end{array}$ & $\begin{array}{l}\text { Cambio } \\
\text { técnico }\end{array}$ & Eficiencia & $\begin{array}{c}\text { Productividad } \\
\text { del trabajo }\end{array}$ & Margen \\
\hline I & $\begin{array}{l}\text { II } \\
\text { III } \\
\text { IV }\end{array}$ & $\begin{array}{l}0,954 \\
0,828 \\
0,324\end{array}$ & $\begin{array}{r}0,767 \\
0,907 \\
0,12\end{array}$ & $\begin{array}{r}0,988 \\
0,0762 \\
0,730\end{array}$ & $\begin{array}{l}0,123 \\
0,000 \\
0,000\end{array}$ & $\begin{array}{l}0,348 \\
0,001 \\
0,000\end{array}$ \\
\hline II & $\begin{array}{l}\text { I } \\
\text { III } \\
\text { IV }\end{array}$ & $\begin{array}{l}0,954 \\
1,000 \\
0,855\end{array}$ & $\begin{array}{l}0,767 \\
0,513 \\
0,011\end{array}$ & $\begin{array}{l}0,988 \\
0,992 \\
0,964\end{array}$ & $\begin{array}{l}0,123 \\
0,900 \\
0,118\end{array}$ & $\begin{array}{l}0,348 \\
0,878 \\
0,403\end{array}$ \\
\hline III & $\begin{array}{c}\text { I } \\
\text { II } \\
\text { IV }\end{array}$ & $\begin{array}{l}0,828 \\
1,000 \\
0,728\end{array}$ & $\begin{array}{l}0,907 \\
0,513 \\
0,063\end{array}$ & $\begin{array}{l}0,762 \\
0,992 \\
0,990\end{array}$ & $\begin{array}{l}0,000 \\
0,900 \\
0,141\end{array}$ & $\begin{array}{l}0,001 \\
0,878 \\
0,626\end{array}$ \\
\hline IV & $\begin{array}{l}\text { I } \\
\text { II } \\
\text { III }\end{array}$ & $\begin{array}{l}0,324 \\
0,855 \\
0,728\end{array}$ & $\begin{array}{l}0,012 \\
0,011 \\
0,063\end{array}$ & $\begin{array}{l}0,730 \\
0,964 \\
0,990\end{array}$ & $\begin{array}{l}0,000 \\
0,118 \\
0,141\end{array}$ & $\begin{array}{r}0,00 \\
0,403 \\
0,626\end{array}$ \\
\hline
\end{tabular}

Fuente: Elaboración propia con información de la Encuesta Nacional de Empleo, Salarios, Tecnología y Capacitación (ENESTYC).

15 Veáse Färe, Grosskopf y otros (1994) y Caves, Christensen y Diewert (1982).

${ }^{16} S$ es un conjunto no vacío, cerrado y convexo y se supone además que tanto los insumos como los productos están libremente disponibles.

${ }^{17}$ La definición formal de las fronteras de producción es: $D^{t}\left(x^{t}\right.$, $\left.y^{t}\right)=\min \left\{\Theta:\left(x^{t}, y^{t} / \Theta\right) S^{t}\right\}, x^{t} \varepsilon \mathrm{R}^{\mathrm{N}}{ }_{+}, t=(1, \ldots, T)$. En otras palabras la distancia $\Theta$ es la relación entre el producto observado y el máximo posible, dado un nivel de insumos. Veáse Färe y Grosskopf (1988).

${ }^{18}$ Las funciones distancia también se pueden expresar en términos de los insumos. Estas funciones miden la máxima disminución posible en los insumos para el mismo nivel de producto. 


\section{Bibliografía}

Amsden, A. (1992): Asia's Next Giant: South Korea and Late Industrialized Countries, Nueva York, Oxford University Press.

Bell, M. y K. Pavitt (1992): Accumulating technological capability in developing countries, Proceedings of the World Bank Annual Conference on Development Economics, Washington, D.C., Banco Mundial.

Cantner, U. y H. Hanusch (2001): Heterogeneity and evolutionary change: empirical conception, findings and unresolved issues, en J. Foster y S. Metcalfe, Frontiers of Evolutionary Economics, Competition, Self-organization and Innovation Policy, Northampton, Edward Elgar.

Carrillo, J. (1993): La Ford en México: reestructuración industrial y cambio en las relaciones sociales, tesis de doctorado en ciencia social, México, D.F., El Colegio de México.

Casas, R., R. de Gortari y M. Luna (2000): University, knowledge production and collaborative patterns with industry, en M. Cimoli, Developing Innovation Systems: Mexico in a Global Context, Londres, Continuum.

Caves, D.W., L.R. Christensen y W.E. Diewert (1982): The economic theory of index numbers and the measurement of input, output and productivity, Econometrica, vol. 50, $\mathrm{N}^{\circ} 6$.

Cimoli, M. (2000): Developing Innovation Systems: Mexico in a Global Context, Londres, Continuum.

Coriat, B. (1992): Pensar al revés, México, D.F., Siglo XXI.

Dillon, W. y M. Goldstein (1984): Multivariate Analysis, Methods and Applications, Nueva York, John Wiley \& Sons.

Dutrenit, G., y M. Capdevielle (1993): El perfil tecnológico de la industria mexicana y su dinámica innovadora en la década de los ochenta, El trimestre económico, vol. 60(3), No 239, México, D.F., Fondo de Cultura Económica, julio-septiembre.

Edquist, Ch. (1992): Technological and organizational innovations, productivity and employment, documento de trabajo, $\mathrm{N}^{\circ} 233$, Ginebra, Programa Mundial del Empleo, Organización Internacional del Trabajo (OIT).

Färe, R. y S. Grosskopf (1988): Fundamentals of Production Theory, Berlín, Springer-Verlag.

Färe, R., S. Grosskopf y otros (1994): Productivity growth, technical progress, and efficiency change in industrialised countries, American Economic Review, vol. 64, Nashville, Tennessee, American Economic Association.

Fransman, M. y K. King (comps.) (1984): Technological Capability in the Third World, Londres, Macmillan.

Hoffman, K. (1989): Technological Advance and Organizational Innovation in the Engineering Industry: A New Perspective of the Problems and Possibilities for Developing Countries, Industry Series Paper No 4, Washington, D.C., Banco Mundial.

Katz, J. (1987): Technology Generation in Latin American Manufacturing Industries: Theory and Case Studies Concerning its Nature, Magnitude and Consequences, Londres, Macmillan.
(1997): New problems and opportunities for industrial development in Latin America, Oxford Development Studies, vol. 25, $\mathrm{N}^{\mathrm{o}}$ 3, Oxford, Taylor \& Francis Group.

Lall, S. (1992): Technological capabilities and industrialization, World Development, vol. 20, $\mathrm{N}^{\circ}$ 2, Amsterdam, Elsevier Science, febrero.

Lall, S. y otros (1994): Technology and Enterprise Development: Ghana Under Structural Adjustment, Londres, Macmillan.

Lundvall, B. (1992): National Systems of Innovation: Towards a Theory of Innovation and Interactive Learning, Londres, Printer Publishers.

Nelson, R. y S. Winter (1982): An Evolutionary Theory of Economic Change, Cambridge, Massachusetts, Harvard University Press.

Pack, H. y L. Westphal (1986): Industrial strategy and technological change: theory versus reality, Journal of Development Economics, vol. 22, № 1, Amsterdam, Elsevier Science.

Pavitt, K. (1984): Patterns of technical change: towards a taxonomy and a theory, Research Policy, vol. 13, N ${ }^{\circ}$ 6, Amsterdam, Elsevier Science.

Romijn, H. (1999): Acquisition of Technological Capability in Small Firms in Developing Countries, Londres, Macmillan.

Schilderinck, J. (1970): Factor Analysis Applied to Developed and Developing Countries, Rotterdam, Rotterdam University Press.

Tabachnick, B. y L. Fidell (2001): Using Multivariate Statistics, Boston, Allyn and Bacon.

Tremblay, P. (1998): Technological Capability and Productivity Growth: An Industrialized/Industrializing Country Comparison, Montreal, Centre Interuniversitaire de Recherche en Analyse des Organisations.

Westphal, L., K. Kritayakirana y otros (1990): The development of technological capability in manufacturing: a macroscopic approach to policy research, en R. Evenson y G. Ranis, Science and Technology: Lessons for Development Policy, Londres, Intermediate Technology Publications.

Westphal, L., y Rhee y G. Pursell (1984): Sources of technological capability in South Korea, en M. Fransman y K. King, Technological Capability in the Third World, Londres, Macmillan.

Wignaraja, G. (1998): Trade Liberalisation in Sri Lanka: Exports, Technology and Industrial Policy, Londres, Macmillan.

(2001): Firm Size, Technological Capabilities and Market Oriented Policies in Mauritius, Discussion papers, $\mathrm{N}^{\circ} 1$, Maastricht, Universidad de las Naciones Unidas (UNU)/Instituto de Nuevas Tecnologías.

Yan Aw, B. y G. Batra (1998): Technological capabilities and firm efficiency in Taiwan (China), World Bank Economic Review, vol. 12, Washington, D.C., Banco Mundial. 